\title{
Percolation Threshold of Conducting Polymer Composites Containing 3D Randomly Distributed Graphite Nanoplatelets
}

\author{
Jing LI and Jang-Kyo KIM* \\ Department of Mechanical Engineering, Hong Kong University of Science and \\ Technology, Clear Water Bay, Kowloon, Hong Kong
}

\begin{abstract}
An improved analytical model is developed based on the average interparticle distance (IPD) concept to predict the percolation threshold of conducting polymer composites containing disc-shaped nanoparticles with high aspect ratios. Two different conditions were taken into account in the model in terms of particle distribution, namely two and three dimensional random orientations. A 10nm interparticle distance is adopted as the electrical conducting criterion according to the tunneling mechanism, and the percolation threshold is estimated as a function of geometric shape of the nanoparticle. A parametric study suggests that the thickness and diameter of fillers are important factors that determine the percolation threshold of conducting nanocomposites. The accuracy and the applicability of the present IPD model are verified by comparing with several existing models and experimental data for graphite nanoplatelet reinforced polymer nanocomposites. It is shown that the current model presents much better agreement with experimental results than existing models.
\end{abstract}

Keywords: Polymer-matrix composites (A); Modeling (B); Electrical properties (B); Graphite nanoplatelets.

\footnotetext{
Corresponding author: Phone) 852-23587207; Fax) 852-23581543; e-mail) mejkkim@ust.hk
} 


\section{Introduction}

Graphite nanoplatelets (GNP), nanoscale conductive filler produced by exfoliating graphite intercalated compound, has attracted significant attention as the low cost alternative to metal- and carbon-based conventional electrically conducting reinforcements for conducting polymer composites, capable of dissipating electrostatic charges and shielding devices from electromagnetic radiation. Previous experimental studies [1 7] suggested that as the conducting filler content gradually increased the composites underwent an insulator-to-conductor transition and a typical percolation behavior was observed. This behavior is attributed to the formation of multiple, continuous electron paths, or conducting network, in the polymer composites with the filler content at or above the percolation threshold. The percolation threshold of GNP reinforced polymer nanocomposites is much lower than the conventional fillers, such as carbon fibres and carbon black, due to the extremely large surface area and high aspect ratio of GNPs.

The percolation theory was applied to explain electrical conductive behavior of composites. Near the percolation threshold, the electrical conductivity of composites follows a power-law relationship [8]:

$$
\sigma=\sigma_{0}\left(V_{f}-V_{c}\right)^{s}
$$

where $\sigma$ is the electrical conductivity of composite, $\sigma_{\mathrm{o}}$ is the electrical conductivity of the filler, $V_{f}$ is the filler volume fraction, $V_{c}$ is the percolation threshold, and $\mathrm{s}$ is a conductivity exponent. Because Equation (1) cannot take into account either of particle 
shape, orientation, polymer-particle interaction or particle dispersion, the value of $s$ is not a constant, but varies with particular composite systems. $s$ and $V c$ have to be determined by curve fitting of experiment results. A recent review of analytical models for electrical conductivity of conductive polymer composites [9] suggests that the majority of existing models dealt with simply shaped fillers, such as rod and close-to-spherical shaped fillers.

Many studies of percolation problems have been based on computational simulations [10 12, 14,15]. Regarding percolation thresholds of fiber reinforced composites, Pike and Seager [10] studied an isotropic system with 2D randomly orientated sticks based on the Monte Carlo method. Balberg and Binenbaum further extended the work to 2D [11] and 3D [12] random anisotropic systems with a preferred fibre orientation, proposing an increased percolation threshold with macroscopic anisotropy and a high dependency of percolation threshold on the aspect ratio of filler. Based on the excluded volume approach [12], the percolation thresholds of polymer composites were studied [13] containing fillers with high aspect ratios, such as micro-scale disc-shaped graphite flakes and carbon fibres with aspect ratios greater than 100. A double inequality was proposed [13] between the upper limit for 3D randomly oriented, infinitely thin disks (or rods) and the lower limit for spheres. As carbon nanotubes become an increasingly important reinforcing material for polymer nanocomposite for many functional and structural applications, theoretical studies have appeared in the literature based on the similar excluded volume approach and the Monte 
Carlo method [14,15]. Another new model was developed recently [16] based on the simple tortuosity-based approach and renormalization group theory to evaluate the geometric factors, such as aspect ratio, orientation and degree of exfoliation, of clay platelets on barrier properties of clay/polymer nanocomposites. A basic hypothesis of the renormalization group theory was that the probability of a cell acting as a barrier was the same on all orders. The results show that the aspect ratio of clay was the most critical parameter in determining the percolation threshold of clay for barrier applications.

In this study, an improved analytical model is proposed based on average interparticle distance (IPD) approach to predict the percolation threshold of polymer nanocomposites containing 3D randomly distributed disc-shaped nanoparticles. The present paper is continuation of our previous studies [6,7] on fabrication, property characterization and applications of conducting GNP reinforced polymer nanocomposites. In this model, 2D and 3D randomly distributed platelets are considered and the influence of geometric shape of platelet is specifically evaluated. The present model is compared with previous models $[13,16]$ as well as experimental data for various GNP nanocomposites made from different matrix materials and varying GNP aspect ratios.

\section{Analysis}

The morphology shown in Figure 1(a) represents a typical individual GNP particle, 
which was modeled as a thin and round platelet in this study. The morphologies of epoxy-based nanocomposites containing $2 \mathrm{wt} \%$ GNP (above the percolation threshold) taken at different magnifications [6] are presented in Figures 1(b) and 1(c). These micrographs suggest that the GNPs of different sizes are randomly dispersed within the matrix. Based on this observation, Figure 1(d) shows a 3D schematic of the conducting network model used in the present study, network formation of which is controlled by many factors, including the volume fraction, aspect ratio and distribution of conductive particles.

To calculate the IPD between adjacent conductive particles, it is assumed that the particles are homogeneously distributed within the matrix and is perfectly bonded with the polymer. It follows then that the composite is divided into cubic elements, each containing one particle in the center, and that the total number of cubic elements is equal to the total number of particles. When the particle has a shape of sphere, as shown in Figure 2(a), the length of the cubic elements, $L$, is given by [17]:

$$
L=D+D_{I P}=\left(\frac{\pi D^{3}}{6 V_{f}}\right)^{\frac{1}{3}}
$$

where $\mathrm{D}$ and $V_{f}$ are the diameter and the volume fraction of particle, $D_{I P}$ is the interparticle distance. Rearranging Eq (2) gives:

$$
V_{f_{-} \text {sphere }}=\frac{\pi D^{3}}{6\left(D+D_{I P}\right)^{3}}
$$

The critical volume fraction of spherical particle corresponding to carbon black or metallic particle-reinforced composites is a function of diameter and the $D_{I P}$ criterion. 
If the conductive particle is in the form of a thin, round platelet with a high aspect ratio, as shown in Figure 2(b), $L$ now becomes:

$$
L=\left(\frac{\pi D^{2} t}{4 V_{f}}\right)^{\frac{1}{3}}
$$

where $t$ is the thickness of platelet. In this case, the IPD is defined as the shortest distance between the neighboring platelets. The orientation state of the platelets can be described in terms of three Eulerian angles $(\theta, \phi, \psi)$, which relate two orthogonal coorinate systems with a common origin. The transition from one coordinate system to the other is achieved by a series of two-dimensional rotations. The angle $\theta$ specifies the rotation of $z$ axis about $y$ axis in the zox plane (See Fig. 2(b)). The angle $\phi$ specifies the relative rotation of $x$ axis about $z$ axis in the xoy plane. The angle $\psi$ specifies the rotation about $z^{\prime}$ axis. In composites, the particles can have an unlimited number of orientations, which can be generalized by a probability distribution function. The probability distribution function, $\rho(\theta, \phi, \psi)$, is introduced to describe the probability of finding a platelet in orientation between $(\theta, \phi, \psi)$ and $(\theta+d \theta, \phi+d \phi, \psi+d \psi) . \quad \rho(\theta, \phi, \psi)$ is used as a weight function at the estimation of the orientation average to describe the orientation state:[18]

$$
\langle F\rangle=\frac{\int_{0}^{2 \pi} \int_{0}^{2 \pi} \int_{0}^{\pi} F(\theta, \phi, \psi) \rho(\theta, \phi, \psi) d \phi d \theta d \psi}{\int_{0}^{2 \pi} \int_{0}^{2 \pi} \int_{0}^{\pi} \rho(\theta, \phi, \psi) d \phi d \theta d \psi}
$$

where the angular brackets denote the orientation average value. $\mathrm{F}$ is the axis-projection function that varies with the orientation state.

For 3D random distribution, the probability distribution function is given by: 


$$
\rho(\theta, \phi, \psi)=\sin \theta
$$

For 2D random distribution, the probability distribution function is given by Dirac delta function:

$$
\rho(\theta, \phi, \psi)=\delta(\theta-0)
$$

To find the geometric relation between $L$ and $D+D_{I P}$, the orientation average of platelets projections along the $\mathrm{x}-, \mathrm{y}-$ and $\mathrm{z}$-axes are calculated as following:

$$
\begin{aligned}
& F_{x}(\theta, \phi, \psi)=D(\cos \psi \cos \phi-\cos \theta \sin \phi \sin \psi)^{2} \\
& F_{y}(\theta, \phi, \psi)=D(-\sin \psi \sin \phi+\cos \theta \cos \phi \cos \psi)^{2} \\
& F_{z}(\theta, \phi, \psi)=D \cos ^{2} \theta
\end{aligned}
$$

After integration, the orientation average of projection tensor now becomes:

$$
\langle F\rangle=\left[\begin{array}{lll}
\frac{D}{3} & \frac{D}{3} & \frac{D}{3}
\end{array}\right]
$$

for 3D random distribution, and

$$
\langle F\rangle=\left[\begin{array}{lll}
\frac{D}{2} & \frac{D}{2} & D
\end{array}\right]
$$

for $2 \mathrm{D}$ random distribution. For convenience, the orientation distribution can also be averaged by the orientation parameters, $f_{p}$ (in-plane orientation parameter) and $f_{a}$ (out-of-plane orientation parameter) [19]:

$$
\begin{aligned}
& f_{p}=2\left\langle\cos ^{2} \phi\right\rangle-1 \\
& f_{a}=\frac{3\left\langle\cos ^{2} \theta\right\rangle-1}{2}
\end{aligned}
$$

$f_{p}$ varies from 0 to $1 . f_{p}=1$ means a perfectly aligned orientation state, whereas $f_{p}=0$ corresponds to an in-plane random orientation (2D), which is often associated with 
extruded composites. In this case, the composite properties are transversely isotropic, and the relevant parameters and L now become:

$$
\begin{aligned}
& \left\langle\cos ^{2} \phi\right\rangle=\frac{1}{2} \\
& L=\frac{1}{2}\left(D+D_{I P}\right)=\left(\frac{\pi D^{2} t}{4 V_{f}}\right)^{\frac{1}{3}} \\
& V_{f_{-} \text {planar }}=\frac{2 \pi D^{2} t}{\left(D+D_{I P}\right)^{3}}
\end{aligned}
$$

The critical volume fraction of 2D random distributed platelets can be calculated using $\mathrm{Eq}(15)$.

The out-of-plane orientation parameter $f_{a}$ ranging form -0.5 to 1 has been widely used to describe the crystalline orientation in semicrystalline polymers. A value of $f_{a}=-0.5$ means that all the elements are perpendicular to $z$-axis and contained within the $x-y$ plane. $f_{a}=1$ corresponds to all elements aligned along the $z$-axis, whereas $f_{a}=0$ corresponds to a uniform distribution at an angle $\theta$, viz., 3D random distribution of platelets for isotropic materials. Similar to the crystalline orientation in semicrystalline polymers, the $3 \mathrm{D}$ random orientation of platelets is incorporated into the model:

$$
\begin{aligned}
& \left\langle\cos ^{2} \theta\right\rangle=\frac{1}{3} \\
& L=\frac{1}{3}\left(D+D_{I P}\right)=\left(\frac{\pi D^{2} t}{4 V_{f}}\right)^{\frac{1}{3}} \\
& V_{f_{-} 3 D}=\frac{27 \pi D^{2} t}{4\left(D+D_{I P}\right)^{3}}
\end{aligned}
$$

Therefore, it can be said that the orientation average values calculated based on these 
two methods, namely the integral of probability distribution function and the orientation parameters, are identical. While the former method has a clearer physical meaning, the latter method is more convenient to use than the former. Eq (18) allows one to estimate the percolation threshold of conducting polymer composites as a function of geometric shape of fillers. It is worth noting that the current cubic-element model (Figure 2(b)) with homogeneously distributed platelets can only be applied to a system containing filler content below the percolation threshold. When using $\mathrm{Eq}$ (18), an electrical conducting criterion has to be introduced so that a maximum $D_{I P}$ is specified corresponding to electron hopping taking place between the adjacent conductive fillers.

\section{Parametric Study}

When the $D_{I P}$ is equal to or less than 10nm, electron hopping happens resulting in a rapid increase in electrical conductivity of composite, according to the quantum-mechanical tunneling mechanism [20]. This phenomenon was reported to be independent of the resistivity of the polymer and be applicable to most polymers, organics and oxides. Thus, $D_{I P}=10 \mathrm{~nm}$ is adopted in this study as the conducting criterion. There are other cases, however, where the $D_{I P}$ should be larger than $10 \mathrm{~nm}$ [21,22]: a multistep electron hopping process was reported in polyethylene and nylon 6,6 matrices, given the critical $D_{I P}$ of $0.7 \mu \mathrm{m}$ for polyethylene [21] and $0.94 \mu \mathrm{m}$ for Nylon 6,6 [22]. Therefore, the $D_{I P}$ criterion in Eq (18) is not a mathematical constant, but represents the influence of properties of matrix material on the electrical behavior of 
composites, which is not negligible in some composite systems.

To verify the IPD model and the applicability of the $D_{I P}$ criterion, a parametric study was performed based on Eq (18) with the $D_{I P}$ criterion varying from $10 \mathrm{~nm}$ to $1 \mu \mathrm{m}$ and the aspect ratio, $\mathrm{D} / \mathrm{t}$, varying from 1 to 5000 (fixed platelet thickness of $10 \mathrm{~nm}$ ) and the results are presented in Figure 3. It is shown that when the critical $D_{I P}$ values were varied between $10 \mathrm{~nm}$ and $1 \mu \mathrm{m}$, the effect of $D_{I P}$ criterion on percolation threshold became negligible as far as the aspect ratio was high enough (i.e. $\mathrm{D} / \mathrm{t} \geq$ about 500). This means that, the $D_{I P}$ criterion can be applied to all kinds of polymer composites containing conducting fillers with a high $\mathrm{D} / \mathrm{t}$. For the nanocomposites reinforced with spherical fillers or platelets with an aspect ratio lower than about 500, the percolation threshold decreased parabolically, depending on $\mathrm{D} / \mathrm{t}$. This observation suggests that the $D_{I P}$ criterion may not be particularly suitable for the composites containing these types of fillers. Therefore, there are two cases: i) when the $D / t \geq 500$, $D_{I P}$ can even be neglected mathematically; ii) when the $\mathrm{D} / \mathrm{t}<500$, the influence of properties of matrix material on the electrical behavior of composites should be taken into account.

The effects of thickness and diameter of platelets on percolation threshold of conducting polymer nanocomposites are shown respectively in Figure 4 and Figure 5. The percolation threshold increased linearly with increasing the platelet thickness, the rate of increase being higher for fillers with a smaller diameter (Figure 4). In contrast, the dependence of percolation threshold on filler diameter was non-linear (Figure 5). 
The percolation threshold decreased parabolically as the filler diameter increased for a given filler thickness. The larger the filler thickness, the larger the variation in percolation threshold. These observations may shed an insight into how one can tailor the shape and geometry of conducting fillers to maximize the benefits arising from the conducting filler and thus to minimize the percolation threshold. It follows then that proper selection of powdering conditions, such as ball milling and ultrasonication, becomes an important task in fabricating conducting polymer composites.

\section{Comparison with Other Models and Experiments}

The percolation threshold values for GNP reinforced polymer nanocomposites with various polymer matrices and different aspect ratios are collected (Table I ) and compared with the theoretical predictions. For the experimental results of percolation threshold reported in weight percentage, conversion to volume percentage was made by assuming the specific gravity of GNP $=2.26 \mathrm{~g} / \mathrm{cm}^{3}$. The diameter and thickness of GNP were determined using several different techniques in the respective papers, including the SEM or TEM measurement, particle size analysis and the estimation from surface area.

These experimental data are compared with theoretical predictions calculated based on several models, including the present model represented by Eq (18), are shown in Figure 6. Noting that the aspect ratios taken from experimental data in Table 1 are all greater than 500 , the influence of $D_{I P}$ on percolation threshold is almost negligible as 
shown in Figure 3. Thus, the $D_{I P}=10 \mathrm{~nm}$ was adopted and the percolation thresholds are plotted as a function of aspect ratio of GNP, instead of the individual geometric parameters, diameter and thickness of GNP.

The previous models used for comparison include the excluded volume model [13] and the renormalization group approach [16]. The excluded volume model proposed by Celzard et al. [13] is given:

$$
1-\exp \left(-\frac{3.6 t}{\pi D}\right) \leq \phi_{c} \leq 1-\exp \left(-\frac{5.6 t}{\pi D}\right)
$$

Lu and Mai's model [16] was originally proposed to predict the barrier properties of polymer-clay nanocomposites with random orientation of platelets:

$$
\phi_{c}=\frac{2.154 t}{D}
$$

Although there were some experimental discrepancies in the estimation of aspect ratio, the experimental results agree well with the prediction based on the present IPD model. The coefficient of determination for the IPD model was 0.903 . The accuracy of the present IPD model was far better than the other two models that tend to underestimate excessively the percolation threshold, being unable to predict the general trend against the aspect ratio of disc-shaped conducting nanofillers.

Meanwhile, for sphere particles with $\mathrm{D}=\mathrm{t}$, the present model based on $\mathrm{Eq}$ (3) gives a percolation threshold of 0.52 when $\frac{D}{D_{I P}}>100$, which is slightly higher than experimental percolation threshold values of 0.428 and 0.4 for $\mathrm{TiC} /$ polyethylene composites [23] and for spherical carbon black polymer composites [24], respectively. It 
is well known that the percolation threshold can be lowered by means of segregation of metallic or carbon black particles [25]. In practice, there are many agglomerates and segregates of reinforcing fillers in the matrix, whereas a homogenous dispersion is normally assumed in the analytical model, which may explain the slight overestimation of $\mathrm{Eq}(3)$.

The above parametric studies and comparisons with experimental data confirm that the present model can be applied to predict percolation thresholds of reinforcements for a wide range of geometric shapes and sizes, including sphere to flat disks with largely different aspect ratios. Nevertheless, there are a few parameters that cannot be taken into account in the present IPD model. They include 1) agglomeration of nanoparticles which are normally observed in many polymer nanocomposites, which is different from the assumption of homogeneous distribution made in the model; 2) presence of micro-flaws or debonding between the matrix and particles, which is different from the assumption of perfect bonding; and 3) irregular shape of conducting fillers, different from the perfect cylindrical shape assumed in the model. These are responsible for the discrepancies between the prediction and experimental data. A well controlled ultrasonication process was critical to producing GNPs with a high aspect ratio while minimizing agglomerates within the matrix [6,7]. Plasma and UV/Ozone treatments $[2,6,7]$ of graphite platelet surface enhanced the interfacial adhesion with polymer matrix. These variations cannot be properly taken into account in the above models. 


\section{Conclusions}

In an effort to predict the electrical behavior of conducting polymer composites containing disc-shaped nanoparticles with high aspect ratios, an improved analytical model based on the average IPD concept is proposed. 2D and 3D random distributions of particles were taken into account in the model. $D_{I P}=10 \mathrm{~nm}$ was adopted as the electrical conducting criterion according to the tunneling mechanism. The aspect ratio of conducting filler was found to be a predominant factor of the nanocomposite percolation threshold. The experimental percolation thresholds of GNP nanocomposites containing various polymer matrices were compared with theoretical predictions based on the present and existing models. The present IPD model agreed better with the published experimental data than existing models, confirming its applicability to predict the percolation behaviour of disc-shaped nano-fillers with high aspect ratios, such as GNP. The parametric study of the present IPD model can shed insights into how one can tailor the geometry of conducting fillers to minimize the percolation threshold of nanocomposite electrical conductivity.

\section{References}

[1] Weng WG, Chen GH, Wu DJ, Yan WL. HDPE/expanded graphite electrically conducting composite. Compos. Interface 2004; 11(2): 131-143.

[2] Fukushima H, Drzal LT. Graphite nanocomposites: structural and electrical properties. Proc. 14th Int. Conf. Compos. Mater. (ICCM-14), San Diego, July 2003. 
[3] Chen GH, Wu DJ, Weng WG, Yan WL. Preparation of polymer/graphite conducting nanocomposite by intercalation polymerization. J. Appl. Polym. Sci. 2001; 82: 2506-2513.

[4] Zheng W, Wong SC. Electrical conductivity and dielectric properties of PMMA/expanded graphite composites. Compos. Sci. Technol. 2003; 63: 225-235.

[5] Chen XM, Shen JW, Huang WY. Novel electrically conductive polypropyrene/graphite nanocomposites. J. Mater. Sci. Let. 2002; 21: 213-214.

[6] Li J, Kim JK, Sham ML, Marom G. Morphology and Properties of UV/Ozone Treated Graphite Nanoplatelet/Epoxy Nanocomposites. Comp. Sci. Techol. 2006; in press.

[7] Li J, Kim JK, Sham ML. Conductive graphite nanoplatelet/epoxy nanocomposites: Effects of exfoliation and UV/ozone treatment of graphite. Scripta Mater. 2005, 53: $235-240$.

[8] Stauffer D, Aharony A. Introduction to percolation theory. London: Taylor and Francis, 1992: 89-113.

[9] Clingerman ML, King JA, Schulz KH, Meyers JD. Evaluation of electrical conductivity models for conductive polymer composites. J. Appl. Polym. Sci. 2002, 83: $1341-1356$.

[10] Pick GE, Seager CH. Percolation and conductivity: a computer study. I. Phys. Rev. B 1974; 10(4): 1421-1434.

[11] Balberg I, Binenbaum N. Computer study of the percolation threshold in a 
two-dimensional anisotropic system of conducting sticks. Phys. Rev. B 1983; 28(7): $3799-3812$.

[12] Balberg I, Binenbaum N, Wagner N. Percolation thresholds in the three-dimensional sticks system. Phys. Rev. Lett. 1984, 52(17): 1465-1468.

[13] Celzard A, McRae E, Deleuze C, Dufort M, Furdin G., Mareche JF. Critical concentration in Percolating systems containing a high-aspect-ratio filler. Phys. Rev. B 1996, 53(10): 6209-6214.

[14] Grujicic M, Cao G, Roy WN. A computational analysis of the percolation threshold and the electrical conductivity of carbon nanotubes filled polymeric materials. J. Mater. Sci. 2004; 39: 4441-4449.

[15] Foygel M, Morris RD, Anez D, French S, Sobolev VL. Theoretical and computational studies of carbon nanotube composites and suspensions: Electrical and thermal conductivity. Phys. Rev. B 2005; 71: 104201-1-104201-8.

[16] Lu C, Mai YW. Influence of aspect ratio on barrier properties of polymer-clay nanocomposites. Phys. Rev. Lett. 2005; 95: 088303-1-088303-4.

[17] El-Tantawy F, Deghaidy FS. Effect of iron oxide on vulcanization kinetics and electrical conductance of butyl rubber composites. Polym Int 2000; 49:1371-1376.

[18] Buryachenko VA, Roy A. Effective elastic moduli of nanocomposites with prescribed random orientation of nanofillers. Comp.: Part B 2005; 36:405-416.

[19] Munson-Mcgee SH, Mccullough RL. Orientation parameters for the specification of effective properties of heterogeneous materials. Polym. Eng. Sci. 1994; 34:361-370. 
[20] Fiuschau GR, Yoshikawa S, Newnham RE. Resistivities of conductive composites. J. Appl. Phys. 1992; 72 (3):953-959.

[21] Wang SF, Ogale AA. Continuum Space Simulation and Experimental Characterization of Electrical Percolation Behavior of Particulate Composites. Comp. Sci. Technol. 1993; 46 (2): 93-103.

[22] Dani A, Ogale AA. Electrical percolation behavior of short-fiber composites: Experimental characterization and modeling. Comp. Sci. Techol. 1996, 56 (8): 911-920. [23] Kataoka M, Masuko T. PTC characteristics of (TiC/polyethylene) conductive composites in relation to their particle-filled structures. Electrical Engineering in Japan $2005 ; 152(2): 1-9$.

[24] Rubin Z, Sunshine SA, Heaney MB, Bloom I, Balberg I. Critical behavior of the electrical transport properties in a tunneling-percolation system. Phys. Rev. B 1999; 59(19): 12196-12199.

[25] Sichel EK. Carbon black-polymer composites. New York: Marcel Dekker,1982: 53-69. 


\section{Figure Captions}

Fig. 1. Scanning electron microphotographs of (a) GNP particle, (b) and (c) random distribution of GNPs within the epoxy matrix; (c) 3D schematic model of GNP conductive network.

Fig. 2. Schematic drawings of the IPD model for (a) sphere fillers and (b) 3D distribution of fillers with high aspect ratios.

Fig. 3. Effect of choosing the $D_{I P}$ criterion on the percolation threshold prediction.

Fig. 4. Effect of thickness of platelet on percolation threshold.

Fig. 5. Effect of diameter of platelets on percolation threshold.

Fig. 6. Comparison of experimental results and theoretical perditions for various GNP reinforced polymer nanocomposites. 


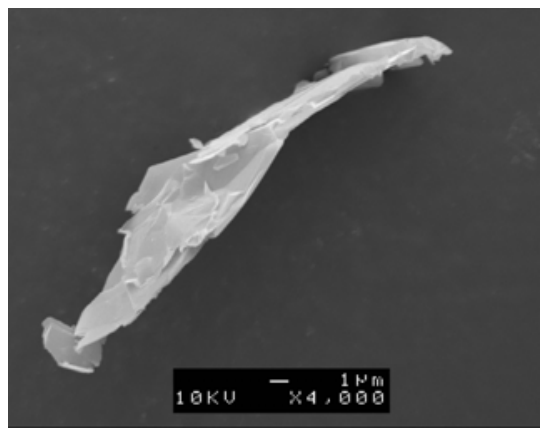

(a)

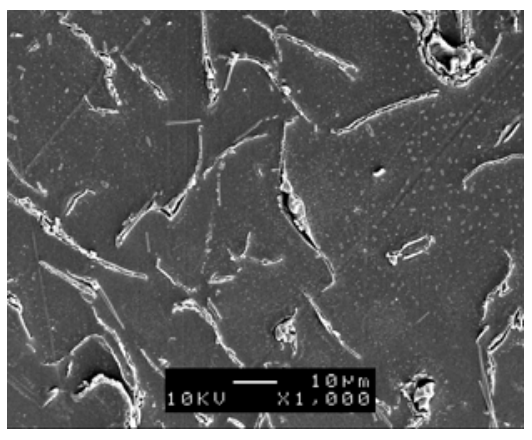

(c)

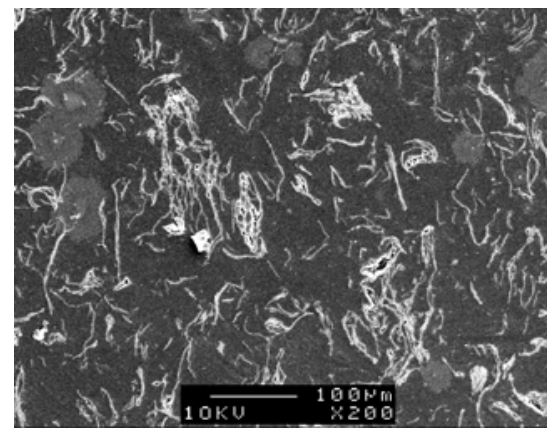

(b)

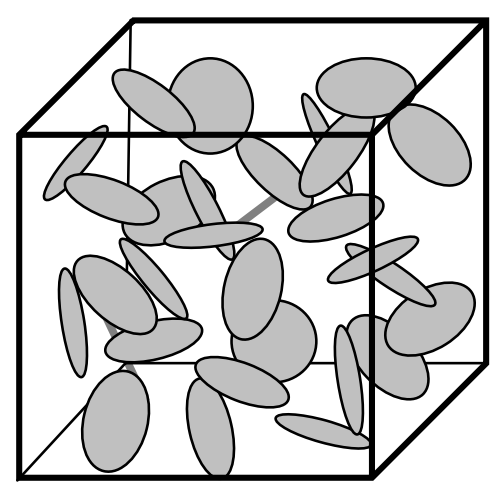

(d)

Fig. 1. Scanning electron microphotographs of (a) GNP particle, (b) and (c) random distribution of GNPs within the epoxy matrix; (c) 3D schematic model of GNP conductive network. 

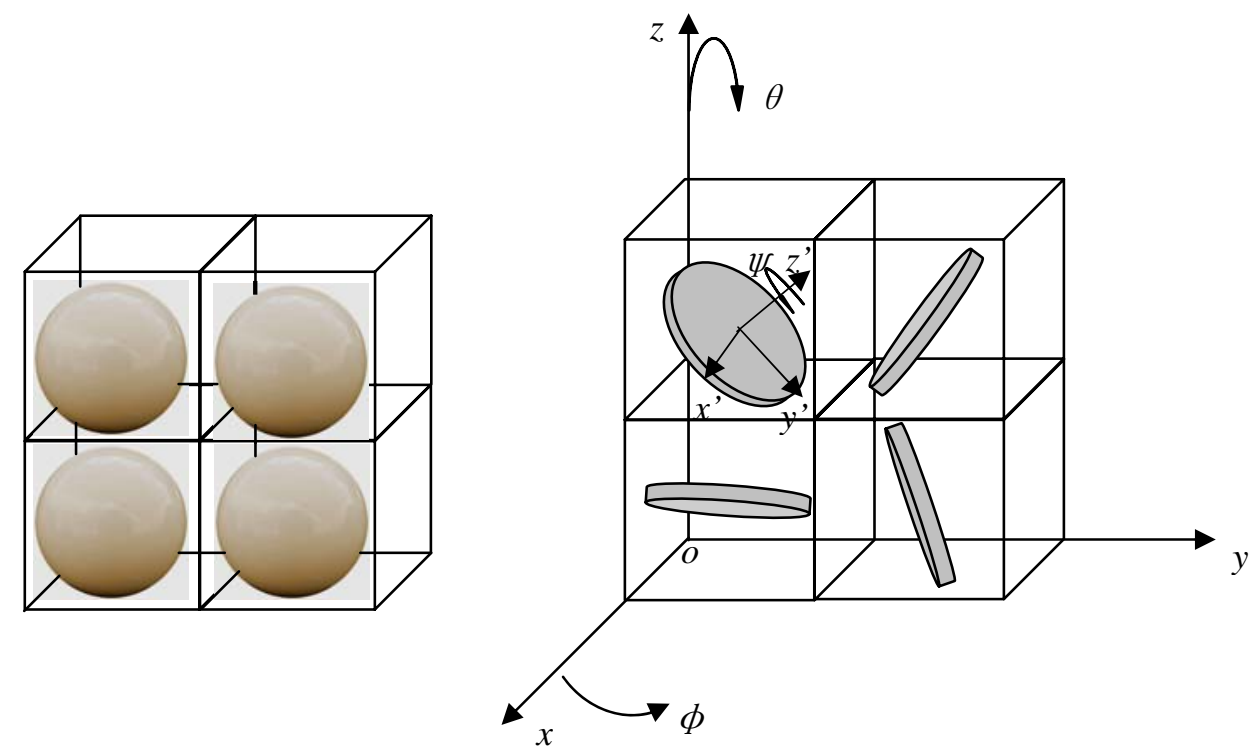

(a)

(b)

Fig. 2. Schematic drawings of the IPD model for (a) sphere fillers and (b) 3D distribution of fillers with high aspect ratios. 


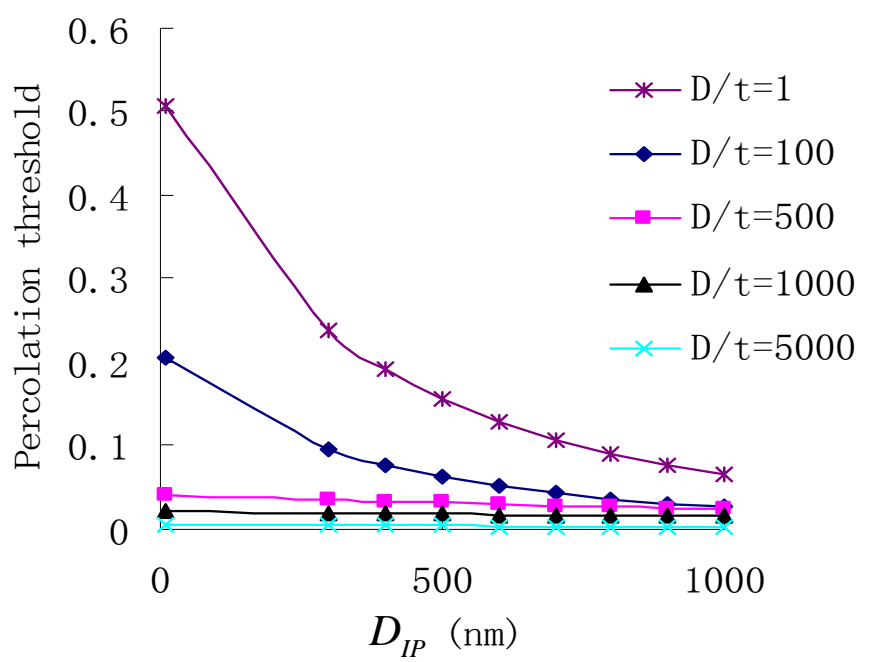

Fig. 3. Effect of choosing the $D_{I P}$ criterion on the percolation threshold prediction. 


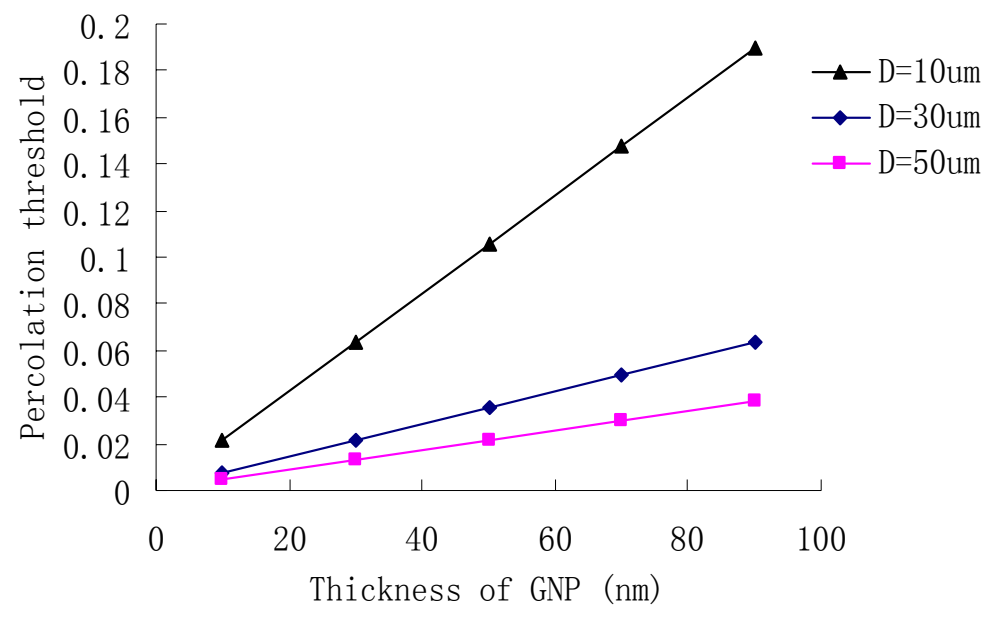

Fig. 4. Effect of thickness of platelet on percolation threshold. 


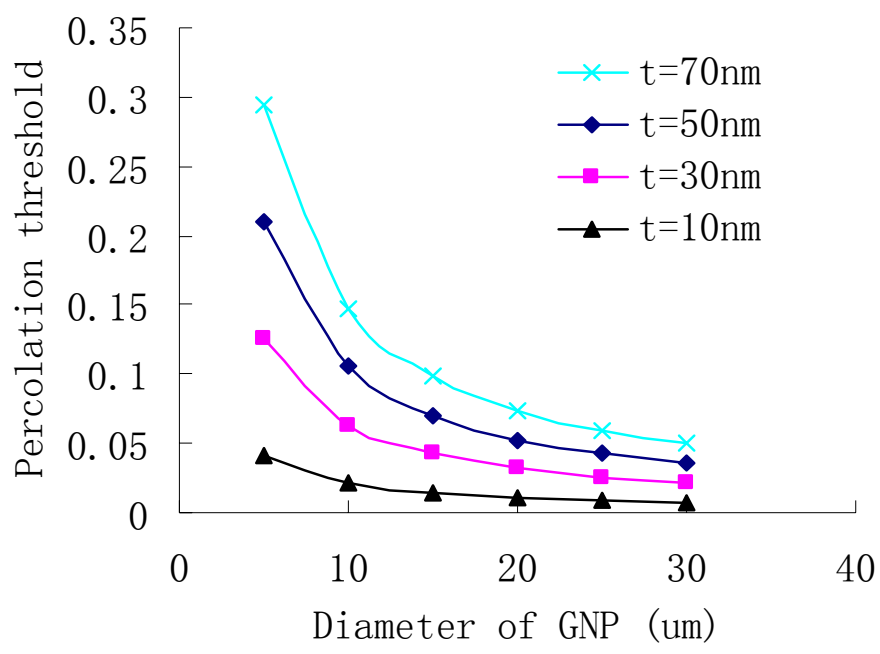

Fig. 5. Effect of diameter of platelets on percolation threshold. 


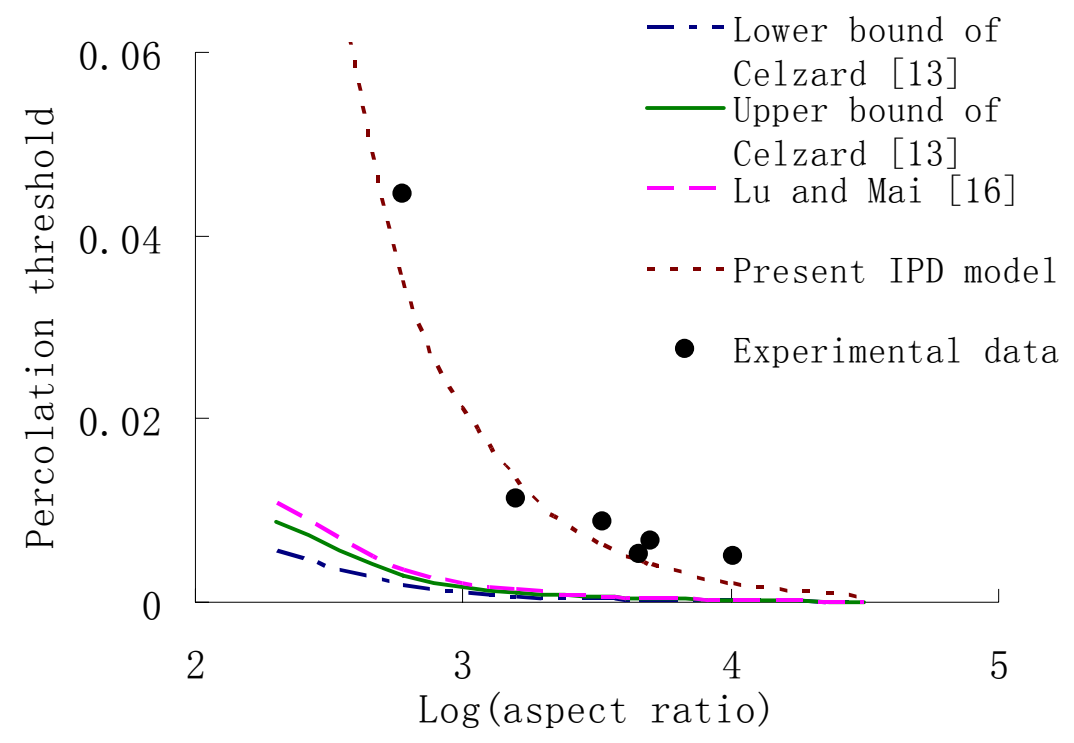

Fig. 6. Comparison of experimental results and theoretical perditions for various GNP reinforced polymer nanocomposites. 
Table 1. Geometric shape factors of the collected experimental data.

\begin{tabular}{|c|c|c|c|c|c|}
\hline Reference & Matrix material & $\mathrm{D}(\mu \mathrm{m})$ & $\mathrm{t}(\mathrm{nm})$ & $\mathrm{D} / \mathrm{t}$ & Percolation threshold \\
\hline Chen et al. [1] & High density polyethylene & 6 & 10 & 600 & $10 \mathrm{wt} \%$ (4.46 vol\%) \\
\hline Fukushima et al. & . [2] Epoxy & 15 & 9.5 & 1579 & $1.13 \mathrm{vol} \%$ \\
\hline Chen et al. [3] & Poly(styrene-methyl mathacrylate) & 100 & 30 & 3333 & $1.8 \mathrm{wt} \%(0.878 \mathrm{vol} \%)$ \\
\hline Zheng et al. [4] & Polymethylmethacrylate & 100 & 22 & 4545 & $1 \mathrm{wt} \%(0.529 \mathrm{vol} \%)$ \\
\hline Shen et al. [5] & Polypropylene & 50 & 10 & 5000 & 0.67 vol $\%$ \\
\hline Li et al. [6] & Epoxy & 46 & 4.5 & 10222 & $1 \mathrm{wt} \%(0.5 \mathrm{vol} \%)$ \\
\hline
\end{tabular}

